\title{
Surgical treatment of hepatic Echinococcus granulosus
}

\author{
Waldemar Patkowski, Maciej Krasnodębski, Michał Grąt, Łukasz Masior, Marek Krawczyk \\ Department of General, Transplant, and Liver Surgery, Medical University of Warsaw, Warsaw, Poland
}

Gastroenterology Rev 2017; 12 (3): 199-202

DOI: https://doi.org/10.5114/pg.2017.70473

Key words: Echinococcosis granulosus, echinococcal cysts, liver resection.

Address for correspondence: Waldemar Patkowski MD, PhD, Department of General, Transplant, and Liver Surgery, Medical University of Warsaw, 1a Banacha St, 2-097 Warsaw, Poland, phone: +48 2259910 00, e-mail: waldemar.patkowski@gmail.com

\begin{abstract}
Introduction: Infections caused by metacestode stage of the Echinococcus granulosus in humans result in disease named cystic echinococcosis.

Aim: To present the outcomes of patients treated surgically for cystic echinococcosis of the liver.

Material and methods: One hundred and nineteen patients treated in the period between 1989 and 2014 due to E. granulosus infection in the Department of General, Transplant, and Liver Surgery, Medical University of Warsaw were selected for this retrospective study. Diagnostic protocol included imaging examinations, i.e. ultrasonography and computed tomography of the abdomen. Blood samples where used to proceed sequential enzyme-linked immunosorbent assay (ELISA) using Em2plus antigen as well as polymerase chain reaction (PCR) to detect $E$. granulosus.

Results: Surgery was the choice for treatment for almost all of the patients (98.3\%). In 40 (34.2\%) patients right hemihepatectomy, in 19 (16.2\%) patients left hemihepatectomy, and in 21 (17.9\%) patients bisegementectomy were performed. Postoperative complications occurred in 4 (3.4\%) patients. In 3 patients biliary fistula requiring endoscopic treatment was observed, and 1 patient had subdiaphragmatic abscess successfully treated with drainage under ultrasound guidance. None of the patients died in the postoperative period, and the 1-, 5-, and 10-year survival rates were $100.0 \%, 90.9 \%$, and $87.9 \%$, respectively.

Conclusions: Surgical treatment of the symptomatic cystic echinococcosis is the modality of choice for $E$. granulosus infection of the liver. Despite substantial development of diagnostic methods and new management opportunities, echinococcal infection still presents a challenge for epidemiologists, pharmacologists, and clinicists.
\end{abstract}

\section{Introduction}

Echinococcosis has been known to humans since antiquity, but it was not until the late $18^{\text {th }}$ century when it was named in the first studies [1]. Echinococcus granulosus is one of the members of the genus Echinococcus of the family Taeniidae. E. granulosus, and together with alveolar Echinococcosis caused by Echinococcus multilocularis, is responsible for the majority of humans' Echinococcus infections [2].

Echinococcus granulosus is a small tapeworm in adult form reaching rarely $7 \mathrm{~mm}$ long. The tapeworm consists of a head (or scolex) and a body (or strobila) with 3 or 4 proglottids. The last one bears the mature eggs. Proglottids contain both male and female organs and serve as reproductive units. In mature form $E$. granulosus parasitises in carnivores (e.g. foxes, wolves, or dogs) intestines, and when its eggs are excreted with faeces the biological life-cycle begins. Eggs containing oncospheres (embryos) released to the environment can be ingested by intermediate hosts (mostly ungulate animals) or humans (aberrant host). After ingestion, the eggs are digested and the oncosphere larva penetrates through the small intestine wall to the circulatory system. Consequently, oncospheres can access any organ, but the majority reach and form cysts in the liver and lungs starting the stage of metacestode (development stage between oncosphere and adult form). Cystic echinococcosis most frequently involves only one organ by forming a solitary cyst. The life-cycle of E. granulosus is complete when the intermediate host is eaten by a definitive host [3-7]. The metacestode growth and formation of a cyst is the beginning of a chronic disease named cystic echinococcosis or hydatidosis [7].

Cystic echinococcosis is a zoonosis with a worldwide geographical distribution. In endemic areas such as South America, Africa, or Central Asia the incidence of cystic echinococcosis can reach up to several dozen cases for every 100,000 people. In Poland the overall incidence count of all types of echinococcosis in 2012 
was 0.073 per 100,000 people. The disease occurs mainly in rural areas with the highest incidence rates observed in the north-eastern part of Poland [5, 8].

Surgical removal of hydatid cysts is the most important treatment for E. granulosus invasion - the mortality ranges from $2.0 \%$ to $4.0 \%$ in properly diagnosed and treated cases. However, it increases to $11.4 \%-22.3 \%$ when the result of treatment is poor or complications occur $[4,9,10]$.

\section{Aim}

The aim of the study was to present the outcomes of patients treated surgically for cystic echinococcosis of the liver.

\section{Material and methods}

Between 1989 and 2014 in the Department of General, Transplant, and Liver Surgery, Medical University of Warsaw 119 patients were hospitalised due to symptomatic Echinococcus granulosus cysts of the liver. The cohort consisted of 73 (61.4\%) women and 46 (38.6\%) men aged from 31 to 72 years. The most typical symptoms were pain (82.3\%) and feeling of fullness or upper abdominal discomfort (65.1\%). Additionally, 54.9\% of patients complained about nausea. Mechanical jaundice was observed in $35.8 \%$ of patients. Hepatomegaly was observed on clinical examination in $74.6 \%$ of patients. Prior to resection, $80.7 \%$ of the patients were treated with antihelmetic therapy (Albendazol).

The diagnostic protocol included imaging examinations, i.e. ultrasonography and computed tomography of the abdomen. Blood samples where used to proceed sequential enzyme-linked immunosorbent assay (ELISA) using Em2plus antigen as well as polymerase chain reaction (PCR) to detect E. granulosus.

\section{Results}

Among 119 patients diagnosed with granular echinococcosis, 2 (1.7\%) were disqualified from surgical treatment due to in-hospital complications - 1 patient

Table I. Types of operations

\begin{tabular}{lc} 
Procedure & Patients \\
\hline Right hemihepatectomy & 40 \\
\hline Left hemihepatectomy & 19 \\
\hline Bisegmentectomy & 21 \\
\hline Non-anatomical liver resection & 28 \\
\hline Cystopericystectomy & 7 \\
\hline Laparotomy and biopsy & 2
\end{tabular}

had haemorrhagic stroke and the second developed myocardial infarction. The remaining 117 (98.3\%) patients were treated surgically. Details of surgical treatment of patients are presented in Table I.

Right hemihepatectomy was performed in 40 (34.2\%) patients, left hemihepatectomy in 19 (16.2\%) patients, and bisegementectomy in 21 (17.9\%) patients. Other operative procedures comprised: non-anatomic liver resection in 28 (23.9\%) patients, cystopericystectomy in 7 (6.0\%) patients, and laparotomy with biopsies taken for histopathological examination (unresectable lesions) in $2(1.7 \%)$ patients.

Major postoperative complications occurred in 4 (3.4\%) patients, including three with biliary fistula requiring endoscopic treatment and one with sub-diaphragmatic abscess successfully treated with percutaneous drainage under ultrasound guidance. None of the patients died in the postoperative period, with 1-, 5 -, and 10 -year survival rates of $100.0 \%, 90.9 \%$, and $87.9 \%$, respectively.

\section{Discussion}

There are four species belonging to genus Echinococcus that can cause diseases in humans: E. granulosus, E. multilocularis, E. vogeli, and E. oligarthrus. Cystic echinococcosis is a chronic disease caused by larval stage of $E$. granulosus and together with alveolar echinococcosis caused by E. multilocularis stands for the majority of human echinococcosis infections [5]. Carnivores, mainly dogs, serve as definitive hosts for E. granulosus. Humans are aberrant immediate hosts as they accidentally consume eggs of adult tapeworms previously excreted by definitive hosts [3]. The beginning period of the infection is asymptomatic and the ingested eggs as oncospheres penetrate through the intestinal wall into the circulatory system of the host. The metacestode stage of the larva begins when the oncosphere settles in one of the host's organs and formation of a cyst is initiated. Liver and lungs account for the majority of hydatid cysts, while infrequent sites of infections include kidneys, brain, heart, and muscles [7].

Two types of $E$. granulosus infections in humans are distinguished; namely primary and secondary cystic echinococcosis. In primary infection (most frequent) the orally ingested eggs reach one of the organs (mainly liver) and form a cyst(s). The secondary form occurs when cyst is ruptured and protoscoleces are released into the abdominal cavity or any neighbouring sites and the process of new infection is initiated $[5,7]$.

Cystic echinococcosis of the liver is characterised by an asymptomatic long lasting phase of the disease. The disease can be asymptomatic permanently and only 
pathological findings after death sometimes confirm the infection. Cysts grow slowly up to $5 \mathrm{~cm}$ per year or stay unchanged for many years. Symptoms vary accordingly to the organ's site of the infection. Symptomatic cysts usually reach $4-5 \mathrm{~cm}$ in diameter. Symptoms of the infection result mainly from pressure of the cyst to adjacent structures and vary from the most common, stomachache, to rare ones such as portal hypertension. The majority of complications of cystic echinococcosis are related to biliary tract, with cystic rupture into bile ducts being the most frequent $[4,5,7,11,12]$.

Diagnosis of hepatic cystic echinococcosis is mainly based upon imaging techniques. The ultrasound screening is still the basis for initial diagnosis (Figure 1). Following initial screening, computed tomography of the abdomen is usually performed (Figure 2). Cysts can be detected using imaging techniques if their size exceeds $1 \mathrm{~cm}$. Ultrasound appearance of the cysts is a basis for Gharbi classification introduced in 1981. Nowadays, the Gharbi's classification has been modified and subsequently the WHO Informal Working Group on Echinococcosis (WHO-IGWE) classification has been introduced. Ultrasound imaging of the cyst can be matched to six distinctive groups proposed by the WHO-IWGE: CL, CE1, CE2, CE3, CE4, and CE5 [13].

The disease can be confirmed after evaluation of specific antibodies. IgG-ELISA should be applied as the first choice test with sensitivity exceeding $90 \%$. The immunoblot or other immunodiagnostic tests could be indicated when IgG-ELISA test is inconclusive. However, in doubtful cases material from puncture or surgical excision needs to be analysed for definitive diagnosis. Material is then examined for DNA tests and histopathology [4].

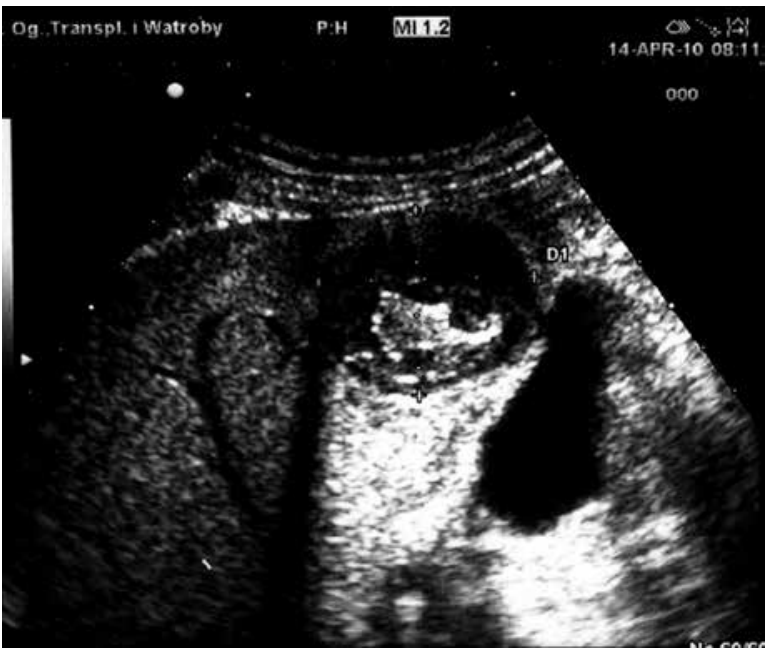

Figure 1. Ultrasound image of Echinococcus granulosus in liver parenchyma
Treatment of cystic echinococcosis is based upon the WHO guidelines and physician's experience. There are no randomised clinical trials to date that could compare different treatment modalities and, accordingly, the strength of recommendations is moderate. Echinococcosis as a rare disease and, in the majority of cases, is treated only in reference centres. Treatment consists of a variety of options. Modalities can be divided into interventional and non-interventional. Interventional methods consist of surgery and percutaneous procedures. Non-interventional management is based on administration of antiparastic drugs. There is also a "watchful waiting" strategy that may be considered in uncomplicated and small lesions.

Surgery should always be considered as a method of choice for symptomatic and complicated cysts. Surgical techniques usually involve anatomical or non-anatomical liver resections, including cystopericystectomy. Surgery as a radical form of treatment provides a chance for cure. There is an ongoing debate on the use of intraoperative solutions to destroy protoscoleces, either by injections of solution into the cysts or using it after accidental cyst rupture to manage the risk of autoinfection [7]. In our department we use hypertonic saline solutions in every case of intraoperative cyst rupture or its risk.

Another modality of interventional treatment is PAIR, which stands for puncture, aspiration, injection, and reaspiration. This ultrasound guided technique is used by some physicians as an alternative to surgery or when surgery is contraindicated. The echinococcal cysts are usually injected with $95 \%$ ethanol $[3,14]$. In our department the invasive treatment modality of choice is surgery, and the PAIR technique is not used.

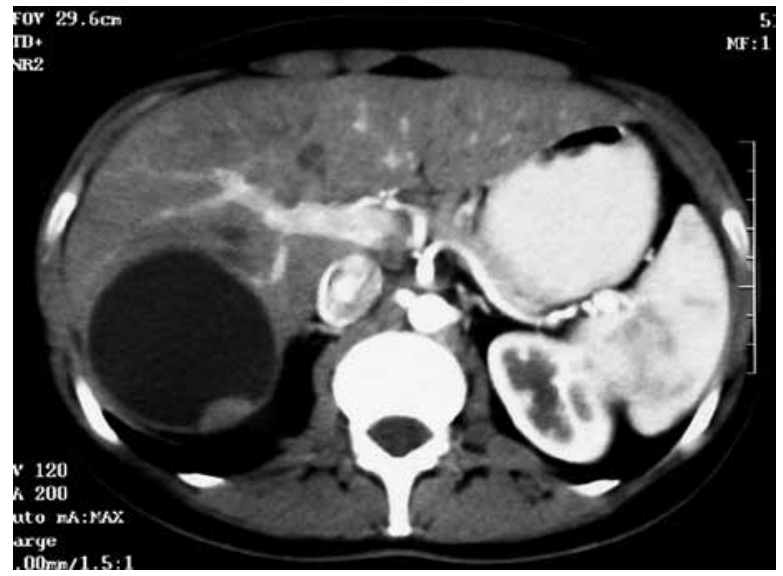

Figure 2. Computed tomography scan of Echinococcus granulosus in liver parenchyma 
Non-invasive treatment with benzimidazoles (mainly albendazol and mebendazol) and praziquantel is used for inoperable patients and also for controlling the disease after surgery. However, the recommendation for using chemotherapeutics also lacks strong evidence as yet. The antiparasitic pharmacotherapy in the postoperative period and after PAIR is usually indicated. The treatment should be continued for at least several years [4].

Surgery remains the therapy of choice for symptomatic cysts. Despite emerging new modalities like PAIR, standard surgery poses acceptable morbidity and recurrence rate with the greatest potential to radical treatment. The study by Georgiou et al. showed that radical surgical procedures are superior to conservative surgical methods like partial cystectomy [15]. The results of surgical treatment in our patients were excellent, with 10 -year survival exceeding $85 \%$. There were no deaths during the postoperative period and the morbidity rate was only $3.4 \%$. The majority of patients underwent radical surgical procedures with right hemihepatectomy being the most frequent (Table I). Liver resection along with cystopericystectomy are radical forms of treating cystic echinococcosis [15]. However, cystopericystectomy in our studied material accounted only for $6 \%$ of all procedures. Cystopericystectomy is a method of choice for many surgical departments [16]. In our opinion liver resection with a broad margin possesses less chance for intraoperative cyst rupture and excellent outcomes in terms of radical treatment.

In recent years more and more studies support laparoscopy as an alternative to "open surgery" for liver resection for benign lesions as well as malignant tumours [17]. A laparoscopic approach for cystic echinococcosis has also been the subject of a number of studies [10, 18]. As long as surgical centres have enough experience in laparoscopic liver resection the laparoscopic modality for echinococcosic cyst treatment is considerable.

\section{Conclusions}

Echinococcal infection usually requires combination of surgical and anti-helminthic treatment. Despite substantial development of diagnostic methods and new management opportunities, echinococcal infection still presents a challenge for epidemiologists, pharmacologists, and clinicists.

\section{Conflict of interest}

The authors declare no conflict of interest.

\section{References}

1. Romig T, Ebi D, Wassermann M. Taxonomy and molecular epidemiology of Echinococcus granulosus sensu lato. Vet Parasitol 2015; 213: 76-84.
2. Craig PS, McManus DP, Lightowlers MW et al. Prevention and control of cystic echinococcosis. Lancet Infect Dis 2007; 7: 385-94.

3. McManus DP, Zhang W, Li J, et al. Echinococcosis. Lancet 2003; 362: 1295-304.

4. Brunetti E, Kern P, Vuitton DA; Writing Panel for the W-I. Expert consensus for the diagnosis and treatment of cystic and alveolar echinococcosis in humans. Acta Tropica 2010; 114: 1-16.

5. Eckert J, Gemmell MA, Meslin FX, Pawłowski ZS. WHO/OIE manual on echinococcosis in humans and animals: a public health problem of global concern World Organisation for Animal Health (Office International des Epizooties) and World Health Organization, 2001. Paris: World Organisation for Animal Health.

6. Larrieu EJ, Frider B. Human cystic echinococcosis: contributions to the natural history of the disease. Ann Trop Med Parasitol 2001; 95: 679-87.

7. Eckert J, Deplazes P. Biological, epidemiological, and clinical aspects of Echinococcosis, a zoonosis of increasing concern. Clin Microbiol Rev 2004; 17: 107-35.

8. Gołąb E, Czarkowski MP. Echinococcosis and cysticercosis in Poland in 2012. Prz Epidemiol 2012; 68: 279-82.

9. Nunnari G, Pinzone MR, Gruttadauria S, et al. Hepatic echinococcosis: clinical and therapeutic aspects. World J Gastroenterol 2012; 18: 1448-58.

10. Yagci G, Ustunsoz B, Kaymakcioglu N, et al. Results of surgical, laparoscopic, and percutaneous treatment for hydatid disease of the liver: 10 years experience with 355 patients. World J Gastroenterol 2005; 29: 1670-9.

11. Siracusano A, Teggi A, Ortona E. Human cystic echinococcosis: old problems and new perspectives. Interdiscip Perspect Infect Dis 2009; 2009: 474368.

12. Frider B, Larrieu E, Odriozola M. Long-term outcome of asymptomatic liver hydatidosis. J Hepatol 1999; 30: 228-31.

13. Working Group WHOI. International classification of ultrasound images in cystic echinococcosis for application in clinical and field epidemiological settings. Acta Tropica 2003; 85: 253-61.

14. Filice C, Brunetti E, Bruno R, Crippa FG; WHO Informal Working Group on Echinococcosis: PAIR Network. 2000. Percutaneous drainage of echinococcal cysts (PAIR-puncture, aspiration, injection, reaspiration): results of a worldwide survey for assessment of its saftey and efficacy. Gut 2000; 47: 156-7.

15. Georgiou GK, Lianos GD, Lazaros A, et al. Surgical management of hydatid liver disease. Int J Surg 2015; 20: 118-22.

16. Junghanss T, da Silva AM, Horton J, et al. Clinical management of cystic Echinococcosis: state of the art, problems, and perspectives. Am J Trop Med Hyg 2008; 79: 301-11.

17. Vavra P, Nowakova J, Ostruszka P, et al. Colorectal cancer liver metastases: laparoscopic and open radiofrequency-assisted surgery. Videosurgery Miniinv 2015; 10: 205-12.

18. Li H, Shao Y, Aji T, et al. Laparoscopic approach for total cystectomy in treating hepatic cystic echinococcosis. Parasite 2014; 21: 65 .

Received: 22.12 .2015

Accepted: 29.02.2016 